\title{
MIE 2017: 1st International Workshop on Multimodal Interaction for Education (Workshop Summary)
}

\author{
Gualtiero Volpe \\ DIBRIS - University of Genova \\ Genova, Italy \\ gualtiero.volpe@unige.it \\ Gabriel Baud-Bovy \\ Italian Institute of Technology \\ Genova, Italy \\ Gabriel.Baud-bovy@iit.it
}

\author{
Monica Gori \\ Italian Institute of Technology \\ Genova, Italy \\ Monica.Gori@iit.it \\ Paolo Alborno \\ DIBRIS - University of Genova \\ Genova, Italy \\ paoloalborno@gmail.com
}

\author{
Nadia Bianchi-Berthouze \\ University College London \\ London, United Kingdom \\ n.berthouze@ucl.ac.uk \\ Erica Volta \\ DIBRIS - University of Genova \\ Genova, Italy \\ erica.volta@edu.unige.it
}

\begin{abstract}
The $1^{\text {st }}$ International Workshop on Multimodal Interaction for Education aims at investigating how multimodal interactive systems, firmly grounded on psychophysical, psychological, and pedagogical bases, can be designed, developed, and exploited for enhancing teaching and learning processes in different learning environments, with a special focus on children in the classroom. Whilst the usage of multisensory technologies in the education area is rapidly expanding, the need for solid scientific bases, design guidelines, and appropriate procedures for evaluation is emerging. Moreover, the introduction of multimodal interactive systems in the learning environment needs to develop at the same time suitable pedagogical paradigms. This workshop aims at bringing together researchers and practitioners from different disciplines, including pedagogy, psychology, psychophysics, and computer science - with a particular focus on human-computer interaction, affective computing, and social signal processing - to discuss such challenges under a multidisciplinary perspective. The workshop is partially supported by the EU-H2020-ICT Project weDRAW (http://www.wedraw.eu).
\end{abstract}

\section{CCS CONCEPTS}

- Applied computing $\rightarrow$ Computer-assisted instruction; Interactive learning environments; $\bullet$ Human-centered computing $\rightarrow$ Human computer interaction (HCI);

\section{KEYWORDS}

Multimodal interactive systems; multimodal interfaces; education; learning technologies.

\section{ACM Reference Format:}

Gualtiero Volpe, Monica Gori, Nadia Bianchi-Berthouze, Gabriel Baud-Bovy, Paolo Alborno, and Erica Volta. 2017. MIE 2017: 1st International Workshop on Multimodal Interaction for Education (Workshop Summary). In

Permission to make digital or hard copies of part or all of this work for personal or classroom use is granted without fee provided that copies are not made or distributed for profit or commercial advantage and that copies bear this notice and the full citation on the first page. Copyrights for third-party components of this work must be honored

For all other uses, contact the owner/author(s).

ICMI'17, November 13-17, 2017, Glasgow, UK

(C) 2017 Copyright held by the owner/author(s)

ACM ISBN 978-1-4503-5543-8/17/11...\$15.00

https://doi.org/10.1145/3136755.3137021
Proceedings of 19th ACM International Conference on Multimodal Interaction (ICMI'17). ACM, New York, NY, USA, 2 pages. https://doi.org/10.1145/ 3136755.3137021

\section{MOTIVATION}

Technology is nowadays increasingly used in many learning environments, including the classroom. Whilst, on the one hand, there exists a vast literature on the usage of multisensory interactive systems for teaching, on the other hand at school the visual channel is often the one most frequently exploited, whereas the other channels are left a marginal role only. Moreover, current technologies for education do not still sufficiently ground on psychophysics and developmental psychology evidence. Recent results from these disciplines (e.g., see [1],[2]) show indeed that children have a preferential sensory channel to learn specific concepts and that the visual signal in not always the more powerful and effective channel for conveying concepts. A pedagogical approach taking into account the best suited modality to teach a specific concept and fully exploiting the potential of multimodal interaction can be highly beneficial for education.

The $1^{\text {st }}$ International Workshop on Multimodal Interaction for Education (MIE2017) aims at investigating how multimodal interactive systems, solidly grounded on psychophysical, psychological, and pedagogical bases, can be designed, developed, evaluated, and exploited for enhancing teaching and learning processes in different learning environments. A particular focus will be on children in the classroom. The workshop represents a unique occasion for researchers and practitioners to meet and discuss multimodal technologies for education under different perspectives, including the pedagogical, psychological, and computational ones, with a strong multidisciplinary approach. A special interest will be on aspects related to Affective Computing (e.g., detection of affective states related to learning) and Social Signal Processing (e.g., how to exploit automated analysis of social interaction in the classroom).

The workshop is partially supported by the EU-H2020-ICT Project weDRAW (http://www.wedraw.eu). weDRAW aims to introduce a new teaching paradigm, based on multisensory interaction, that exploits the most effective sensory channel in children. Specifically, the workshop will start from the knowledge acquired in the first year of weDRAW, and will be open to new perspectives and experiences. 


\section{TOPICS}

The program encompasses two invited talks by prof. Rita Cucchiara and prof. Stephen Brewster, and 14 presentations (5 long papers, 5 short papers, and 4 posters/demos) covering a broad variety of topics around multisensory technologies for education.

More in detail, the accepted contributions can be grouped into three major clusters: papers that address the pedagogical and psychophysical bases multisensory technology for education needs to ground on, papers that presents technological research in the area (e.g., how to capture and analyze information from the different sensory channels), and papers that focus on studies concerning technology-enhanced learning in specific domains (e.g., music, maths, computational thinking, and so on).

Concerning the pedagogical and psychophysical bases, one paper illustrates a pedagogical framework to design multimodal and multisensory serious-games for children. Another paper focuses on a psychophysical analysis of children's angle perception while freely performing full-body movements in space. These two contributions are exemplar of how studies in pedagogy and psychophysics can provide guidance to technological development.

Regarding the technological framework, several papers address different areas of research in multisensory technology: one paper introduces the use of haptic force-feedbacks in educational settings to enhance learning experience; another one presents a review of the state-of-the-art of affective computing in the educational context; others more specifically describe machine learning techniques to analyze students' engagement using different measures of attention, exploiting facial expressions, gaze direction, and eyes movement related to personality traits.

As for case studies in specific areas, three papers address learning to play a music instrument: the first one explores the effects of feedback technologies on violin learning in novice students, the second one presents a machine learning model to predict bowing velocity during violin performances, and the third one proposes a machine learning approach to classify left-hand fingering in studying violin. One paper presents a LEGO $^{\circledR}$ based multimodal interface for children, where elements from the music domain and from computer programming are mixed to convey some basic aspects of computational thinking. Two contributions address serious-games to teach mathematical concepts to primary school children. Finally, one paper introduces automatic generation of feedback to improve social competency in job interviews using an online learning environment.

\section{FUTURE PERSPECTIVES}

We believe the topics the MIE2107 workshop addresses may have a strong impact on future perspectives for the research community, for industry, and the society. The investigation of the role different modalities and multimodal integration have on the teaching and learning processes can provide a deeper understanding of such processes and support new pedagogical paradigms. This knowledge can inform industrial development of novel products for education, grounded on scientific bases and exploiting multimodal technology. Such a synergy between scientific research and industrial development can lead to more effective and pleasant approaches to learning with a beneficial effect on the society. We think that MIE2017 can contribute to these future perspectives with a broad discussion of current research and an analysis of future research and application challenges e.g., in terms of a collection of scientifically grounded guidelines for designing, developing, and evaluating multimodal interactive systems for education.

\section{ACKNOWLEDGMENTS}

The MIE2017 workshop is partially supported by the EU-H2020-ICT Project weDRAW. weDRAW has received funding from the European Union's Horizon 2020 Research and Innovation Programme under Grant Agreement No. 732391. We thank the ICMI workshop chairs Miriam Redi, Martin Pielot, and Mohamed Chetouani for their kind assistance. We also thank the ICMI publication chairs, the invited speakers, and the authors. Finally, we would like to address a special thanks to the MIE2017 reviewers for their excellent work in reviewing the papers submitted to the workshop during summer.

\section{REFERENCES}

[1] Maeve M. Barrett and Fiona N. Newell. 2015. Task-Specific, Age Related Effects in the Cross-Modal Identification and Localisation of Objects. Multisensory Research 28, 1-2 (2015), 111-151.

[2] Monica Gori, Michela Del Viva, and Giulio Sandini. 2008. Young Children Do Not Integrate Visual and Haptic Form Information. Current Biology 18, 9 (2008), 694-698. 